\title{
Gli “eredi" di Calvino negli anni ottanta: Andrea De Carlo e Daniele Del Giudice
}

Les « héritiers » de Calvino dans les années quatre-vingt: Andrea De Carlo et Daniele Del Giudice

Calvino's heirs in the eighties: Andrea De Carlo and Daniele Del Giudice

\section{Sabina Ciminari}

\section{OpenEdition}

\section{Journals}

Edizione digitale

URL: http://journals.openedition.org/cei/463

DOI: $10.4000 /$ cei.463

ISSN: 2260-779X

\section{Editore}

UGA Éditions/Université Grenoble Alpes

\section{Edizione cartacea}

Data di pubblicazione: 15 mars 2012

Paginazione: 163-181

ISBN: 978-2-84310-222-6

ISSN: 1770-9571

Notizia bibliografica digitale

Sabina Ciminari, «Gli "eredi" di Calvino negli anni ottanta: Andrea De Carlo e Daniele Del Giudice», Cahiers d'études italiennes [Online], 14 | 2012, online dal 15 septembre 2013, consultato il 26 mars 2021. URL: http://journals.openedition.org/cei/463 ; DOI: https://doi.org/10.4000/cei.463 


\title{
GLI “EREDI” DI CALVINO NEGLI ANNI OTTANTA: ANDREA DE CARLO E DANIELE DEL GIUDICE
}

\author{
Sabina Ciminari \\ Université de Savoie
}

Un discorso sulla questione degli "eredi", quale è quello che ci proponiamo di fare, comporta una riflessione critica su una possibile linea Calvino-Del Giudice-De Carlo e, all'interno di essa, sul ruolo di maestro che Italo Cavino, più o meno consapevolmente, ha avuto. La dichiarazione, in apertura del nostro intervento, di una tale posizione, non è esente da possibili contestazioni, soprattutto se esse assumono, come punto di partenza, l'esame delle ultime opere dei due eredi in questione, Andrea De Carlo e Daniele Del Giudice, quale punto d'arrivo di un'evoluzione narrativa che, a partire dagli anni Ottanta, ha attraversato il decennio successivo fino ad arrivare ai nostri giorni.

Orizzonte mobile (2009) e Durante (2008) ${ }^{\mathrm{I}}$, da questo punto di vista, sono due romanzi che hanno in comune ben poco, e rappresentano gli esiti di percorsi narrativi che non solo hanno preso strade diverse, ma che sono il risultato di una riflessione che si è svolta in momenti e seguendo tempi diversi. De Carlo ha pubblicato al ritmo di circa un romanzo ogni due anni; Del Giudice è tornato alla ribalta delle cronache letterarie italiane - rifiutandosi fra l'altro di concorrere nel 2009 al Premio Strega, per il quale era considerato uno dei favoriti - a distanza di oltre vent'anni dal suo ultimo romanzo.

Eppure, il discorso cambia se ci si concentra sugli anni Ottanta, che segnano i loro inizi, e in particolare sui romanzi d'esordio: De Carlo e Del Giudice aprono, infatti, il decennio con due opere che restano sotto molti punti di vista fra le loro migliori prove letterarie: Treno di panna e

I. Al momento in cui questo articolo è stato scritto è annunciata la pubblicazione dell'ultimo romanzo di De Carlo, Leielui (Milano, Bompiani, 20Io), che non è stato quindi preso in considerazione nella nostra analisi critica. 
Lo stadio di Wimbledon, pubblicate rispettivamente nel I98I e nel I983, per i tipi dell'Einaudi.

Una prospettiva simile di rilevazione di una linea di filiazione comune per questi romanzi è stata, fra l'altro, già ipotizzata da altri: si pensi in particolare a Filippo La Porta che, pur dopo aver parlato di una narrativa caratterizzata dall'assenza di padri e maestri dichiarati, nella sua Nuova narrativa italiana ${ }^{2}$ inserisce questi autori (insieme a Mario Fortunato, Andrea Canobbio e Dario Voltolini) nel primo capitolo della sua ricostruzione critica, dal titolo suggestivo Il Calvino dimezzato, con la motivazione che "Calvino $[\ldots]$ sarà uno dei pochi "maestri" italiani riconosciuti dagli scrittori di questo periodo» ${ }^{3}$.

Se la posizione di La Porta ci sembra condivisibile, tanto più lo è se messa in relazione a quell'idea "del lavoro artigianale della scrittura» che ha accompagnato Calvino, il cui impegno editoriale, prova e manifestazione di essa, è considerato ad esempio da Remo Ceserani come la testimonianza più eloquente del suo essere un postmoderno sui generis ${ }^{4}$.

Da un punto di partenza che si colloca a cavallo fra queste due posizioni critiche ha origine il nostro discorso sugli anni Ottanta. Ragioni che sono anche editoriali, infatti, e comunque interne a un lavoro, in Einaudi, che comportava anche la promozione di giovani autori, oltreché un'adesione evidente e dichiarata a un tipo di narrativa e a un modo di intendere il racconto, spingono Calvino a diventare e a essere riconosciuto dalle voci narrative che esordiscono in quegli anni come un punto di riferimento, e nello specifico come un maestro di visione narrativa, questione sulla quale - nel contesto di un panorama letterario europeo segnato dall'école du regard - andava ragionando proprio in quegli anni. Nel 1983 ad esempio guarda alla letteratura europea contemporanea rilevando in essa l'insistenza sull'elemento visivo:

In Francia [...] il problema della "cosa in sé" ha continuato a contrassegnare la ricerca letteraria [...]. Ma credo che l'ultima parola non sia stata ancora detta. Recentemente in Germania Peter Handke ha scritto un romanzo basato interamente sui paesaggi. E anche in Italia un approccio visivo è l'elemento comune di alcuni degli ultimi nuovi scrittori che ho lettos.

2. F. La Porta, La nuova narrativa italiana. Travestimenti e stili di fine secolo, Torino, Bollati Boringhieri, 1995 (riedito in una nuova edizione nel 1999). Di una «scia di Calvino» parla anche Fabio Pierangeli, nella sua Ultima narrativa italiana (1983-2000), Roma, Studium, 2000, affiancando i nomi di Del Giudice e di De Carlo a quello di Gianni Celati, definito un «altro "discepolo" di Calvino".

3. Ibid., p. II.

4. R. Ceserani, Raccontare il postmoderno, Torino, Bollati Boringhieri, 1997, p. I7I sgg.

5. I. Calvino, Mondo scritto e mondo non scritto (1983), in Id., Saggi 1945-1985, a cura di M. Barenghi, t. II, Milano, Mondadori, coll. «I Meridiani», 1995, p. I873. 
Da Calvino quindi prenderà avvio il nostro discorso, con un sommario esame di quello su cui si era concentrato prima dell'improvvisa scomparsa, nel 1985. Si passerà quindi all'esame degli esordi di De Carlo e Del Giudice, che si collocano lungo una linea di sviluppo e di continuazione della sua ricerca. Concluderemo con uno sguardo all'evoluzione narrativa dei due scrittori, la cui produzione letteraria arriva fino ai nostri giorni, per dimostrare quanto i loro percorsi possano essere considerati, in parte, corrispondenti al "carattere binario» ${ }^{6}$ del pensiero di Calvino, e quanto al tempo stesso - è il caso soprattutto di Del Giudice - essi possano far pensare a un ideale proseguimento della sua attività letteraria.

L'improvvisa scomparsa di Italo Calvino, nel settembre del 1985 , lascia orfano il pensiero critico e letterario degli anni Ottanta, tanto più perché interrompe bruscamente un percorso di riflessione che era ancora in piena costruzione. Si pensi al lavoro su quelle che sono comunemente conosciute come le Lezioni americane, sulle quali lo scrittore era impegnato quando è stato colto da un ictus. Le Norton Lectures, le «sei proposte per il prossimo millennio» alle quali si era dedicato nell'estate di quell'anno («Siamo nel 1985: quindici anni appena ci separano dall'inizio d'un nuovo millennio" ${ }^{7}$, si legge in apertura, secondo una prospettiva quindi che si vuole intrinsecamente legata a un presente che corrisponde al cuore degli anni Ottanta, e al tempo stesso si apre al futuro), sono un'importante opera saggistica, restata incompiuta. Pubblicate nel I988, prendono la forma con la quale sono conosciute oggi, delle lezioni magistrali a cui Calvino stava affidando la sua idea del valore letterario: Leggerezza, Rapidità, Esattezza, Visibilità, Molteplicità. Cinque valori ai quali andrebbe aggiunto il sesto, la Consistency, che avrebbe dovuto chiudere il discorso: la congruità alla quale la sua scrittura, pur attraversata da quelle che sono definibili tentazioni postmoderne, sembra votata.

Tentazioni che, a livello narrativo, si manifestano nel romanzo che chiude gli anni Settanta, ed è al tempo stesso tutto proteso verso gli anni Ottanta: Se una notte d'inverno un viaggiatore (1979), vero caso non solo letterario ma editoriale dell'epoca, il cui successo coglie di sorpresa il suo stesso autore. Vicini al gioco combinatorio che anima quest'opera, e impregnati da un'attenzione alla ricerca e alla descrizione scientifica - in genere molto marginale nel panorama della nostra storia letteraria, ma che non a caso

6. Si richiama a esso, fra gli altri, Mario Barenghi nell'introduzione alla raccolta dei saggi di Calvino nella sopracitata collezione dei «Meridiani».

7. I. Calvino, Lezioni americane. Sei proposte per il prossimo millennio (1988), in Id., Saggi 1945-I985, op. cit., t. I, p. 629. 
segna le pagine di un autore come Del Giudice - sono anche gli esperimenti delle Cosmicomiche, la cui pubblicazione inizia negli anni Sessanta e che appariranno nel 1984 in una edizione definitiva dal titolo Cosmicomiche vecchie e nuove.

Ai fini del nostro discorso è interessante ricordare che la voce narrativa di Calvino è rimasta ferma al signor Palomar (1983), l'ultimo progetto narrativo che porta a termine, nato sulle pagine del Corriere della Sera nel 1975. Palomar, il protagonista-alter ego dell'autore, è il nome dell'acuto osservatore che si muove «in cerca d'un'armonia in mezzo a un mondo tutto dilaniamenti e stridori», e diventa l'ultima incarnazione letteraria della passione di Calvino per la descrizione: «È da parecchio tempo che cerco di rivalutare un esercizio letterario caduto in disuso e considerato inutile: la descrizione», scrive in una presentazione di Palomar, rimasta inedita fino alla sua raccolta nei «Meridiani ${ }^{8}$. Palomar cerca di fare ordine in tutto questo, e quello che ne risulta «non è un libro di racconti, né di meditazioni». Sono, queste, le parole di Daniele Del Giudice, che ai tempi di Palomar fa risalire i suoi rapporti con Calvino, e che alla raccolta dedica un articolo intitolato significativamente L'occhio che scrive, in cui insiste sulla visività come cifra distintiva della narrazione:

Ed è proprio questa esperienza della visività, spinta al suo punto limite, che determina la forma del libro: un libro cioè di descrizioni. [...] In Palomar, la descrizione, proprio perché storia ininterrotta di una costituzione del mondo e dell'io mediati dallo sguardo, e dunque ricerca di un sentire, di un comportamento, di una saggezza, rimescola in sé il romanzesco che normalmente è altrove; e come descrizione, credo, diventa una forma nuova, autonoma e potenziata di racconto ${ }^{9}$.

Ritorneremo su questa nuova forma di racconto che Del Giudice individua e alla quale si ispirerà: per il momento ci basti insistere sul fatto che Palomar presenta una smania catalogatrice che sembra costituire un pendant del lavoro compilativo che Calvino sta facendo a livello saggistico: all'inizio degli anni Ottanta infatti Calvino raccoglie alcuni dei suoi interventi critici in Una pietra sopra (1980) e in Collezione di sabbia (1984).

Questo per quanto riguarda l'edito. Fra i libri pensati e non realizzati che la morte improvvisa ha lasciato allo stato di progetto, c'è il volume Passaggi obbligati, con il quale Calvino intendeva riunire quanto aveva scritto, e si proponeva di scrivere, di autobiografico (e che sarà pubblicato

8. I. Calvino, Romanzi e racconti, edizione diretta da C. Milanini, a cura di M. Barenghi e B. Falcetto, t. II, Milano, Mondadori, coll. «I Meridiani», I994, p. I404.

9. D. Del Giudice, «L'occhio che scrive», Rinascita, 20 gennaio 1984, raccolto in Italo Calvino. Enciclopedia: arte, scienza e letteratura, a cura di M. Belpoliti, Milano, Marcos y Marcos, coll. «Riga, 9", I995, pp. I76-I79. 
parzialmente nel 1990 nella Strada di San Giovanni), e l'idea di una raccolta sui Cinque sensi - un altro tema che è ben vivo nell'immaginario dei suoi eredi - di cui ci rimangono i tre racconti di Sotto il sole giaguaro (I986).

Accanto alla saggistica e alla narrativa, a cavallo fra gli anni Settanta e l'inizio del decennio successivo, Calvino prosegue la sua attività editoriale. I libri degli altri, la scelta di oltre trecento lettere - sul numero definito da Maria Corti «sconcertante» di cinquemila - tratte dall'epistolario editoriale di Calvino, reca in epigrafe l'ormai famosa citazione: «il massimo del tempo della mia vita l'ho dedicato ai libri degli altri, non ai miei. E ne sono contento» ${ }^{\mathrm{IO}}$.

Il passaggio dai suoi libri a quelli degli altri è quello che ci proponiamo, a nostra volta, di fare. Il percorso che affronteremo si vuole, quindi, rigorosamente unitario: scelte narrative fanno luce su prese di posizioni editoriali di Calvino, e viceversa. Il lavoro editoriale dello scrittore ligure permette infatti di estrapolare importanti elementi della sua poetica, e di far luce sulle sue idee critiche e teoriche ${ }^{\text {II }}$. In questo senso, l'inizio degli anni Ottanta è particolarmente significativo, e vede l'affacciarsi, sulla scena editoriale e letteraria, di due giovani autori, Andrea De Carlo, classe 1952, e Daniele Del Giudice, classe 1949, i cui esordi narrativi sono patrocinati da Calvino stesso.

I loro primi romanzi possono essere ricondotti all'incirca a uno stesso momento, nel contesto di una Einaudi che sta attraversando un momento di grave crisi aziendale - Calvino stesso, che amava definirsi «editorialmente monogamo", nel 1984 accetterà l'offerta di Garzanti, presso cui in quell'anno pubblica Collezione di sabbia e Cosmicomiche vecchie e nuove-, e corrispondono al primo atto di una produzione narrativa capace di durare nel tempo: ancora oggi, entrambi gli autori sono attivi sul mercato editoriale (certo in modo molto diverso, con un ritmo più cadenzato Del Giudice, e con uno più incalzante De Carlo) con buone risposte, rispettivamente, di critica e di pubblico. Due scrittori che ora ci sembrano molto

Io. I. Calvino, I libri degli altri. Lettere I947-I98I, a cura di G. Tesio, con una nota di C. Fruttero, Torino, Einaudi, I991. Le parole di Calvino, poste in epigrafe alla raccolta, sono tratte da un'intervista a Marco d'Eramo apparsa su Mondoperaio nel 1979. Maria Corti ha dato una prima lettura de I libri degli altri nelle pagine intitolate Il lettore misantropo, raccolte in Calvino \& l'editoria, a cura di L. Clerici e B. Falcetto, Milano, Marcos y Marcos, 1993, pp. 167-175.

II. "Calvino editore non è separabile dal Calvino tutto intero, e questo rende difficile scriverne», così Giulio Bollati inizia il suo intervento, nel convegno del 1990 dedicato ai legami fra Calvino e l'editoria, e questa illuminante affermazione deve a nostro avviso accompagnare, molto più di quanto ciò non avvenga, ogni riflessione critica sulla figura di Calvino.» (G. Bollati, "Calvino editore», Micromega, n. I, I99I, poi raccolto in Calvino \& l'editoria, op. cit., p. I.) 
distanti l'uno dall'altro, ma che hanno avuto un debutto quasi comune, all'interno dello scenario editoriale dei primi anni Ottanta.

In questo periodo, infatti, i tempi sembrano maturi per un rilancio della narrativa italiana: i successi di Se una notte d'inverno un viaggiatore (1979) e del Nome della rosa (1980), per non dimenticare il libro-evento della fine degli anni Settanta, Porci con le ali, che nel 1976 segnala una forte richiesta di narrativa giovane, incoraggiano le case editrici a puntare sulla letteratura italiana. È come se si fosse pronti a rischiare di più, e anzi la narrativa italiana, soprattutto se rappresentata da autori che non superano i trent'anni, costituisce un sicuro investimento per un tipo di editoria, quella cosiddetta di "terza generazione", dominata dal best seller e da tirature medie che corrispondono quasi al doppio (siamo nell'ordine delle 8.500 copie) rispetto a quelle dei decenni successivi (nel 2003, ad esempio, le tirature medie viaggiano intorno alle 4.800 copie, fatta eccezione, ovviamente, per operazioni editoriali di investimento su alcuni titoli considerati particolarmente forti). Un'editoria libraria che è segnata ormai sempre di più - e così sarà fino ai nostri giorni - da un prevalere della promozione sulla capacità di produrre titoli, da una politica della novità, spesso effimera, alla quale si sacrificano il catalogo e, con esso, la possibilità che le opere e gli autori più significativi hanno di durare.

In questo contesto si possono rilanciare editorialmente alcuni degli scrittori più rappresentativi della generazione del dopoguerra, presentandoli come dei casi letterari - si pensi a Morante, a Pasolini, allo stesso Calvino - e, al contempo, una nuova leva di scrittori si può affermare sul mercato. Alcuni esordi positivi (come Altri libertini di Pier Vittorio Tondelli per Feltrinelli, e De Carlo, appunto, per Einaudi) creano infatti un clima favorevole alla pubblicazione di altri debutti - clima, questo, che si protrae per tutto il decennio ${ }^{12}$.

Nello specifico, è in questo contesto che l'Einaudi intraprende una strategia concertata di lancio di giovani scrittori, che godono dell'appoggio di un patrocinatore - modalità di promozione, questa, che altre case editrici si affretteranno a prendere ad esempio - come Calvino: personaggio non solo autorevole, all'epoca, ma consacrato dal successo di Se una notte d'inverno un viaggiatore, e quindi ormai collocabile oltre il ristretto circolo degli

I2. Fra i numerosi lavori che vanno tenuti presenti, nel ricostruire lo scenario dell'editoria libraria degli anni Ottanta che fa da sfondo al nostro discorso, si tengano in considerazione almeno i seguenti testi: S. Tani, $I l$ romanzo di ritorno. Dal romanzo medio degli anni Sessanta alla giovane narrativa degli anni Ottanta, Milano, Mursia, 1990; G. C. Ferretti, Storia dell'editoria letteraria in Italia. 1945-2003, Torino, Einaudi, 2004; G. Ragone, L'editoria in Italia. Storia e scenari per il XXI secolo, Napoli, Liguori, 2005. 
addetti al lavoro, nonché da sempre impegnato a seguire con attenzione le sperimentazioni dei giovani e gli sviluppi della narrativa contemporanea.

I «Nuovi Coralli» accolgono prima il più giovane Andrea De Carlo con Treno di panna, nel 198r. Due anni dopo esce La stadio di Wimbledon di Del Giudice. Entrambi recano la presentazione di Italo Calvino.

La giovinezza è tante cose, anche una particolare acutezza dello sguardo che afferra e registra un enorme numero di particolari e sfumature; un'insaziabilità degli occhi che bevono lo spettacolo del mondo multicolore ingigantito come attraverso la lente d'un teleobbiettivo e lo depositano in fotogrammi miniaturizzati nella memoria. È questa la giovinezza che Andrea De Carlo racconta: la storia d'un ragazzo italiano piombato a Los Angeles non sa neanche lui perché e che cerca d'arrangiarsi con mestieri occasionali, è seguita attraverso tutto quello che capita nel raggio dei suoi occhi attenti e imperturbabili $[\ldots]^{13}$.

Calvino, in questa ormai famosa quarta di copertina che accompagna la prima edizione del romanzo, dimostra di seguire con attenzione un personaggio che, proprio come il suo Palomar, sembra dominato dall'acutezza dello sguardo: "occhi attenti e imperturbabili», e al contempo insaziabili, sono definiti quelli del protagonista. Un modo di guardare che è segnato dalla "giovinezza»: un termine editorialmente accattivante, certo, ma che qui significa anche altro, in quanto determina la qualità di un modo di raccontare che è anche un modo di stare al mondo. Calvino parla di giovane narrativa, e si serve della categoria per promuovere un romanzo, ma lo fa seguendo una sua particolare idea di letteratura. Prosegue, infatti:

Oggi ci imbattiamo spesso in una scrittura giovanile in cui domina lo sfogo degli stati d'animo, il rimescolamento interiore, il dramma esistenziale: nati da un'esigenza di sincerità assoluta questi testi di solito non ci dànno che un repertorio di clichés e di espressioni generiche: schermi verbali che nascondono più di quanto non esprimono. Andrea De Carlo è tutto il contrario: proiettato come è sul «fuori» non è escluso che egli riesca a farci intravedere qualcosa del «dentro». [...] Non a caso Andrea De Carlo, prima di affrontare il romanzo, ha cercato di esprimersi con la fotografia; scrivendo sembra voler sostituire la penna all'obbiettivo fotografico [...].

Certo, non è un segreto il rapporto problematico che Calvino intrattiene con l'autobiografia, e con un racconto di sé che si fa solo «sfogo», appunto. De Carlo è considerato emblematico di una tendenza inversa, di un "non racconto di sé”, per la qualità della sua narrazione che si limita a raccogliere l'altro da sé, e a descrivere e trasfigurare, con una tecnica apparentabile

I3. Questo risvolto, e quello del romanzo di Del Giudice, oltre ad essere leggibili nelle rispettive prime edizioni, sono oggi raccolti nel volume Il libro dei risvolti, a cura di C. Ferrero, Torino, Einaudi, 2003, insieme a tutti i testi firmati da Calvino o a lui attribuibili. La raccolta si vuole esemplificativa della scrittura editoriale alla quale Calvino si è dedicato sin dal suo ingresso in Einaudi, nel 1947. 
a quella fotografica, l'esperienza autobiografica. Non è un caso, quindi, che Calvino sottolinei l'esperienza precedente di De Carlo - fotografo in America - prima del suo passaggio a scrittore e autore di un romanzo di formazione moderno che racconta le peregrinazioni e le avventure di un fotografo, Giovanni Maimeri, sullo sfondo di una vacua Los Angeles fatta "di freeways e di parcheggi, nei ristoranti italiani coi camerieri messicani, nelle ville delle dive del cinema».

De Carlo stesso ha sottolineato in più occasioni la qualità fotografica della sua narrazione: «Soprattutto all'inizio forse, nei primi libri, era più evidente una forte influenza anche della fotografia», afferma in un'intervista del 1995, che potremmo citare come esemplificativa di un suo costante ricorrere alle immagini e alla fotografia per descrivere il suo modo di narrare. E prosegue:

La capacità di lettura della fotografia, soprattutto di un tipo di fotografia che a me affascinava molto e tuttora affascina, è legata a certe forme pittoriche come potrebbe essere l'iperrealismo americano in cui c'è questa specie di ultradefinizione nei particolari che è reale, ma anche leggermente alterata, perché non è proprio una riproposizione realistica, assolutamente. È comunque una deformazione del reale. Però ti permette di arrivare al dettaglio più sottile ${ }^{14}$.

Una dei suoi ultimi lavori, inoltre, è stata la realizzazione di un progetto capace di unire narrativa e fotografia, associandole alla sua passione per la musica: Dentro "Giro di vento". Musiche, foto, parole, ha infatti accompagnato l'uscita del romanzo Giro di vento (2004), con un CD di composizioni scritte e interpretate da De Carlo e di fotografie commentate. Infine, a vent'anni da Due di due, il suo più grande successo della fine degli anni Ottanta, nella presentazione editoriale preparata per la nuova edizione del testo, De Carlo ha sottolineato la centralità della visibilità nel suo processo creativo: «Come mi succede ogni volta che inizio un romanzo, sono partito da un'immagine. Quando l'immagine finalmente arriva, è come una fotografia sfuocata, ma diventa più nitida ogni volta che torno a guardarla. Poco a poco i dettagli si delineano, volti e gesti diventano leggibili» ${ }^{15}$. Non è un caso, quindi, che nel parlare di arte - e De Carlo, si noti, ha disegnato alcune delle copertine dei suoi libri - la sua preferenza dichiarata vada alla pittura iperrealista. La descrizione e la visualità, siano esse proprie alla pittura o alla fotografia, giocano quindi un ruolo centrale nel racconto.

I4. C. Klettke (a cura di), «A colloquio con Andrea De Carlo», Italienisch, n. 17, 1995, raccolto in A colloquio con... Interviste con autori italiani contemporanei, a cura di C. Lüderssen e S. A. Sanna, Firenze, F. Cesati, 2004, p. 184 .

15. A. De Carlo, Due di due, Milano, Bompiani, 2009 [1989]. 
Si legga, a titolo esemplificativo, il seguente passo tratto dal suo romanzo d'esordio:

Era una sensazione quasi fisica: la scomparsa graduale della mia presenza in termini visualmente percepibili. Mi pareva di essere sullo specchio ribaltabile di una macchina fotografica mentre chi la tiene in mano gira l'anello della messa a fuoco su una distanza ravvicinata. I miei contorni si dissolvevano progressivamente. [...] Cercavo di scoprire fino a che punto potevo avvicinarmi senza essere notato. [...] Ma questo stato invisibile non durava mai molto: a tratti la lente girava verso di me e venivo improvvisamente a fuoco, stagliato sullo sfondo di tavoli e sedie ${ }^{16}$.

Pagine analoghe, come fossero le avventure di un fotografo di calviniana memoria ${ }^{17}$, si ritrovano anche nell'esordio narrativo di Del Giudice, che prende il titolo da una scena finale del romanzo ${ }^{18}$. Anche qui, come in De Carlo, un protagonista si muove in una sorta di viaggio di formazione che si compie fra Trieste e l'Inghilterra: luoghi percorsi sulle tracce di Bobi Bazlen, figura emblematica e quasi mitica dello scrittore che non ha scritto. A Londra per incontrare l'ultima compagna di Bazlen, Ljuba, il giovane protagonista si ferma in visita allo stadio di Wimbledon e, mentre si trova al museo, osserva che "Tutti gli oggetti sono isolati dalle passioni, in una loro perplessità, come le foto" (p. II2). Poi, guardando il campo dal palco centrale, si ferma a pensare al significato della ricerca e del viaggio intrapresi:

Nemmeno la densità del luogo è un aiuto, anzi. In fondo tra poco avrò l'ultima occasione, e dovrei trovare qualcosa che mi portasse di colpo al perché lui non ha scritto; ma ho solo pensieri confusi, e un senso di lontananza da quella domanda come da un vortice di acume, o di rigore, o di ironia per compensare, o di angoscia paralizzante, o non so (p. II3).

Sembra arrivato vicino alla soluzione, eppure questa gli sfugge. Lo scrittore che non ha mai scritto, preferendo la vita alla letteratura, lo spinge a compiere, a sua volta, un atto di rinuncia alla parola in favore della visione:

Qualunque frase è contro il panorama. Vorrei solo vedere, e sentire; e per la prima volta è spiacevole, proprio adesso, non poter fotografare una visione di insieme, o un particolare che conta solo per me. Prendo un quaderno dalla borsa, per disegnare; nel movimento ho l'impressione di una piccola forma nera sulla destra. Lo sguardo

I6. A. De Carlo, Treno di panna, Torino, Einaudi, coll. «Nuovi Coralli», I981, pp. 67-68.

17. Il riferimento, ovvio, è al non-fotografo poi fotografo, della presenza e infine dell'assenza, Antonino Paraggi, protagonista dell'Avventura di un fotografo, testo che, nato in forma di saggio nel 1955, fa la sua apparizione in forma narrativa nel volume Gli amori difficili (Torino, Einaudi, 1970).

I8. D. Del Giudice, Lo stadio di Wimbledon, Torino, Einaudi, coll. «Nuovi Coralli», I983. Le citazioni si riferiscono all'ultima edizione attualmente in circolazione, del 1996, che si chiude sulla Nota di Italo Calvino, che nella prima edizione era collocata in quarta di copertina. 
torna indietro da sé: sulla panca al di là dei gradini c'è un cinturino che pende a terra, e un astuccio con un naso pronunciato, e dentro può esserci solo una cosa al mondo: una macchina fotografica (ibid.).

Quasi come se arrivasse in soccorso dell'osservatore, si materializza un apparecchio fotografico, ma il protagonista indugia troppo e un altro visitatore, più lesto, glielo sottrae, mentre lui assiste impassibile agli eventi: «Non faccio nulla; aspetto che le cose accadano, come sempre. Occupo il tempo scegliendo già qualche immagine» (p. II4). La scelta delle immagini, preliminare alla fissazione delle stesse, attraversa la scrittura narrativa di Del Giudice, permeata da un insistere sulla visione ${ }^{19}$ che sembra apparentarsi, in questa sua prima forma narrativa, alla pagina di De Carlo.

Nella presentazione che Calvino fa dello Stadio di Wimbledon, ritroviamo inoltre un richiamo alla sfera semantica della giovinezza: «Questo romanzo racconta d'un giovane che si interroga su un certo personaggio, a una quindicina d'anni dalla sua morte; e va a ricercarne gli amici e le amiche di gioventù, ora molto anziani». Questo l'incipit, mentre verso la fine si legge:

A un certo punto del suo itinerario (o già in principio?) il giovane ha fatto la sua scelta: cercherà di rappresentare le persone e le cose sulla pagina, non perché l'opera conta più della vita, ma perché solo dedicando tutta la propria attenzione all'oggetto, in un'appassionata relazione col mondo delle cose, potrà definire in negativo il nocciolo irriducibile della soggettività, cioè se stesso.

Cosa ci annuncia questo insolito libro? La ripresa del romanzo d'iniziazione d'un giovane scrittore? $\mathrm{O}$ un nuovo approccio alla rappresentazione, al racconto, secondo un nuovo sistema di coordinate? (La «carta di Mercatore» è una delle immagini(hiave) ${ }^{20}$.

19. Su questo elemento ricorrente della poetica di Del Giudice si sono soffermati alcuni degli interventi critici sull'autore, che si sono succeduti sempre più numerosi, a partire dalla fine degli anni Ottanta. Si vedano alcuni titoli di saggi e di interviste, in cui la descrizione e la visione emergono come chiavi interpretative privilegiate: Il tempo del visibile nell'Atlante di Daniele Del Giudice, D. Del Giudice a colloquio con S. Bertolucci, T. Gaddi, A. Pastorino e G. L. Saraceni, in Palomar. Quaderni di Porto Venere, n. I, primavera 1986, pp. 67-96; A. Dolfi, «Sul filo dell'iride. Daniele Del Giudice e la geometria della visione», Esperienze letterarie, n. 19, I994, pp. 19-30; P. Antonello, "La verità degli oggetti. La narrativa di Daniele Del Giudice fra descrizione e testimonianza», Annali d'italianistica, n. 23, 2005, pp. 2II-23I. Al contempo, la critica più attenta ha individuato l'origine di questa poetica dell'immagine dell'autore proprio nella sua relazione con Calvino: fra i vari contributi in cui questa relazione è citata, si veda almeno P. Daros, «Image et représentation chez Italo Calvino et Daniele Del Giudice», Chroniques Italiennes. Italo Calvino, les mots, les idées et les rêves, n. 75-76, 2005, pp. 77-96.

20. Calvino aveva presentato il romanzo in Einaudi nel corso della riunione editoriale del Is dicembre 1982 , qualificandolo come "Libro molto semplice come lettura diretta, ma allo stesso tempo con molto spessore e di qualità notevole», con il titolo di Carta di Mercatore (cfr. C. Ferrero (a cura di), Il libro dei risvolti, op. cit., p. 217): come ricordato da Del Giudice, la perplessità dimostrata in casa editrice verso questo titolo lo porterà a optare per Lo stadio di Wimbledon. 
L'attenzione all'oggetto, al mondo delle cose, la rappresentazione come centro della narrazione. Anche qui, come nelle pagine di Treno di panna, quello che Calvino sembra apprezzare maggiormente è la qualità dello sguardo dell'autore. È questa la linea della giovane narrativa che Calvino vuole promuovere: "gli occhi che scrivono", potremmo dire parafrasando il titolo dell'intervento che Del Giudice dedica a Palomar.

Calvino sembra seguire con attenzione, quindi, in questi primi anni Ottanta, una scrittura che potremmo definire fotografica; cinematografica, anche. Non è un caso, infatti, che entrambi questi romanzi d'esordio siano stati adattati per il grande schermo, anche se in tempi molto diversi. Treno di panna è l'unico film che De Carlo ha realizzato come regista, nel I988, curandone anche la sceneggiatura ${ }^{21}$. E dichiarata è l'influenza che la sua scrittura deve al cinema, come ha avuto modo di ricordare lo scrittore stesso:

Il cinema e la fotografia mi hanno influenzato profondamente, prima ancora di avere la possibilità di lavorare in quei campi. Quando ho cominciato a scrivere ho capito che un romanziere può utilizzare alcune tecniche visive nella costruzione delle proprie storie: per esempio il close-up, o al contrario la visione a distanza come attraverso un teleobiettivo, oppure il flash-back, il flash-forward, lo slow-motion, l'accelerazione, il taglio di fotogrammi. Basta che chi scrive si conceda la stessa libertà che il cinema ha sempre avuto nei confronti della letteratura, attingendo a trame, personaggi, temi, atmosfere ogni volta che ne aveva bisogno ${ }^{22}$.

Mentre Le stade de Wimbledon, che resta distribuito solo in Francia, è portato sullo schermo nel 2002 dal regista e attore Mathieu Amalric. Questi, a causa dello smarrimento della sceneggiatura preparata per girare il film, si servirà - stando almeno alle sue dichiarazioni - dell'edizione francese del romanzo come unica forma di sceneggiatura ${ }^{23}$.

2I. Il film è stato presentato alla Mostra internazionale d'arte cinematografica di Venezia, nel I988. Giovanni Maimeri è impersonato da Sergio Rubini, che sullo schermo diventa un musicista, da fotografo quale era nel romanzo, e si muove sullo sfondo di New York (la Los Angeles originaria). Fra gli altri interpreti: Carol Alt e Cristina Marsillach, mentre Ludovico Einaudi, che firma le musiche, fa anche una breve apparizione.

22. "Intervista ad Andrea De Carlo, a cura di G. De Luca», Italica, n. 84, 2007, pp. 838-842, anche on-line all'indirizzo <http://findarticles.com/p/articles/mi_hbI432/is_4_84/ai_n2944242I > [consultato il 20 ottobre 2OII].

23. Il film è accompagnato da Malus, diciotto minuti di racconto-documentario che il regista fa della storia del suo incontro con il romanzo e con il suo autore. Le stade de Wimbledon è interpretato da Jeanne Balibar, che offre una personificazione femminile al protagonista de Lo stadio di Wimbledon. Di particolare interesse, ai fini del nostro discorso, un momento del documentario in cui Del Giudice mostra ad Amalric le immagini - schizzi, polaroid - che ha fatto della sua visita londinese: dalla casa di Lijuba allo stadio di Wimbledon. «C'est curieux - dice Del Giudice nell'intervista, sottolineando la centralità dell'immagine come punto di partenza della sua operazione narrativa - un cahier, quatre photos qui deviennent un film, qui deviennent un livre. C'est presque comme un délit. Il y a des éléments en charge, des épreuves, le prove.» 
Il visualismo di Calvino trova quindi come oggetti privilegiati di lettura e di promozione editoriale due romanzi che, seguendo la sua linea di ricerca, hanno come punto di partenza l'immagine. E se ormai è celebre l'affermazione che nel I96o rivolge al suo amico François Wahl - «l'unica cosa che vorrei insegnare è un modo di guardare, cioè di essere al mondo» ${ }^{24}-$, così come è stata studiata la centralità della metafora ottica nel complesso della sua produzione narrativa e saggistica ${ }^{25}$, resta ancora da indagare la linea calviniana di ricerca intorno a quell'interesse, pur fondamentale, verso «i libri degli altri», che ha costituito gran parte dell'impegno intellettuale di Calvino.

Così la descrizione scientifica, l'acutezza della visione, il rapporto fra letteratura e cinematografia, la descrivibilità del mondo in termini linguistici, sono gli elementi che attraggono Calvino e che lo portano a promuovere le opere prime di De Carlo e Del Giudice. La visibilità, prima di tutto, che è anche una delle sue proposte per il nuovo millennio, oltre a essere uno dei sensi ancora in corso di elaborazione per il suo progetto di raccolta.

Nelle lettere che ha scritto a De Carlo, ad esempio, questo dato emerge chiaramente, e proprio sull'affinamento di questa "visione" convergono i suoi consigli. Gli scrive ad esempio l'I novembre 1980, mentre lo rassicura sulle sue "doti naturali» di scrittore, e al tempo stesso lo invita a vigilare sulla necessità di un'economia espressiva:

Caro Andrea,

Ho letto Treno di panna. Trovo che hai una grande capacità di osservazione e di resa di sensazioni sottili e complesse. La parte in cui descrivi il lavoro nel ristorante è secondo me la più ricca come scrittura e come vivacità. Alle volte sei troppo ricco di dettagli: ti manca il senso dell'economia espressiva e della sintesi.

La cosa che più mi intriga nel tuo romanzo è che mentre ci viene offerta questa profusione di sensazioni oggettive, non viene mai detto niente o quasi niente dei pensieri e stati d'animo dei personaggi, soprattutto del protagonista. S'intravvede solo un gran senso di vuoto. Direi che questa zona di silenzio o d'opacità, forse da te calcolata ma forse no, sia la vera forza poetica del romanzo. L'interesse a leggerlo è interesse a veder vivere delle persone, dei giovani, e a cercar di capire cosa vogliono, cercar di capirlo insieme a loro, mentre neanche loro lo sanno! Hai saputo trattenerti dal cercare di rappresentare i loro pensieri, cosa che di solito riesce fasulla, con parole prese a prestito ${ }^{26}$.

24. Lettera di I. Calvino a F. Wahl, Torino, I dicembre I960, raccolta in I. Calvino, I libri degli altri, op. cit., pp. 350-35I e successivamente in Lettere. 1940-1985, a cura di L. Baranelli, introduzione di C. Milanini, cronologia a cura di M. Barenghi e B. Falcetto, avvertenza di L. Baranelli, Milano, Mondadori, 20oo, coll. «I Meridiani», pp. 668-669.

25. Di fondamentale importanza, in proposito, il bel saggio di M. Belpoliti, L'occhio di Calvino, Torino, Einaudi, 1996 (riedito in una versione ampliata nel 2006) che ha contribuito a ispirare la nostra riflessione.

26. I. Calvino, I libri degli altri, op. cit., p. 627. 
Il I6 dicembre 1980, in un'altra lettera, insiste sulla necessità di acquisire «una maggiore padronanza dei mezzi letterari», e gli rivolge delle proposte di correzione che sembrano declinarsi tutte in relazione alla qualità visiva della sua scrittura:

Per esempio frasi del tipo «Era una sensazione quasi lancinante» (p. 35): lancinante è un aggettivo molto forte; se ci metti quasi gli togli forza. Ma la sensazione lancinante dovrebbe averla il lettore già da quello che tu rappresenti, senza bisogno che tu la definisca. Nella tua impostazione oggettiva devi rendere sensazioni precise e non commentare sensazioni con aggettivi.

Alle volte usi parole che non esistono e come invenzioni linguistiche non valgono molto «fantasizzavo» invece di «fantasticavo» a p. 80. Oppure dettagli inutili che se passano veloci rendono la concretezza della visione; ma alle volte sono proprio troppo minuziosi e ci si chiede perché: per esempio a p. 247: «Siamo scesi con movimenti quasi paralleli; abbiamo chiuso le portiere nello stesso momento» ${ }^{27}$.

«Un paio di lettere»: questo è quanto sembra restare a testimonianza della relazione fra Calvino e De Carlo. Come quest'ultimo ha ricordato di recente, infatti, il suo non è stato un rapporto con l'uomo-Calvino. De Carlo non nasconde il fatto di averlo «visto solo due o tre volte, altrettante gli ho parlato al telefono, e ci siamo scritti un paio di lettere», e la riconoscenza per la decisione - presa probabilmente in accordo con Natalia Ginzburg - di pubblicarne il primo romanzo, quando lui era un giovane sconosciuto (due romanzi rimasti nel cassetto, e numerosi rifiuti per Treno di panna):

Malgrado questo - aggiunge - senza di lui forse non avrei mai pubblicato il mio primo romanzo. Forse addirittura non ne avrei più scritti. Quello che ho imparato da lui è passato attraverso i suoi libri, fin da quando ho letto Il barone rampante alla scuola media e sono rimasto colpito dalla limpidezza e dalla precisione della sua lingua, così lontana dalla bolsa tradizione letteraria italiana ${ }^{28}$.

È un'eredità esplicita, quella che De Carlo dichiara, ed è un'eredità che passa per una lezione di stile che Calvino ha trasmesso a tutta una generazione di nuovi narratori, e che resta valida anche se questi hanno poi scelto strade diverse.

De Carlo, ad esempio, pubblica per Einaudi Uccelli da gabbia e da voliera nel 1982. È sempre introdotto da Calvino ma ora la quarta di copertina è anonima. Il risvolto, questa volta, sembra segnato da un tono appena più

27. Ibid., pp. 629-630.

28. "Intervista inedita ad Andrea De Carlo", a cura di R. Petito, 8 aprile 2003, in R. Petito, Andrea De Carlo e la narrativa degli anni Ottanta. Guida alla lettura, con intervista inedita ad Andrea De Carlo, Venezia, Edizioni Studio LT2, 2005, p. I49. 
distaccato: la storia è quella di «un giovane che sembra sappia vivere solo la propria non adesione al mondo che lo circonda», e il riferimento, ovvio, va al primo romanzo. Si legge infatti: "Treno di panna, il libro che ha rivelato Andrea De Carlo, ci aveva dato i fotogrammi d'una scoperta giovanile del mondo. In Uccelli da gabbia e da voliera s'aggiunge l'elemento romanzesco col suo suspense». La chiusura della presentazione, in cui si parla di «un romanzo d'amore e d'azione, che non tradirà la nostra attesa» ${ }^{29}$, con il tono marcatamente promozionale che la caratterizza, sembra precorrere quella via del (buon) romanzo di consumo che, in alternativa al romanzo d'autore, De Carlo sembra progressivamente imboccare.

La svolta, che alcuni critici fanno risalire a Macno $(1984)^{30}$, forse non a caso coincide con il cambiamento di editore: De Carlo lascia un'Einaudi in piena crisi e approda a Bompiani, l'editore che ancora oggi ne accoglie le pubblicazioni. E una strada decisamente più facile che fa di lui un autore di grandi tirature, che si contraddistingue per una facilità di scrittura tale da permettergli di pubblicare un romanzo in media ogni due anni. Dopo le prime prove, e in particolare dopo l'esordio narrativo di Treno di panna, che pure ha contribuito a catalizzare l'interesse editoriale per l'opera prima di giovani autori ${ }^{31}$, e le cui pagine hanno fatto parlare - con la cifra particolare, quasi alienata, del suo modo di descrivere, individuata per primo da Calvino - di una via italiana all'école du regard francese, De Carlo sembra aver scelto la strada di una narrativa più di consumo, che si fa quasi manierista nella ripetitività delle soluzioni adottate e nell'adozione di uno stile ben riconoscibile, che lascia spazio a poche sorprese.

È evidente infatti l'insistere su un tipo di personaggi e di situazioni: i protagonisti sono sempre giovani uomini, che si pongono in modo alternativo alla realtà e alla società che li circonda; vivono storie di amicizia e, intrecciate a esse, di amore, che permettono loro di crescere, di trovare una via al passaggio verso la maturità. Le situazioni narrative si fanno prevedibili, seppur raccontate con talento e in modo sapiente, per cui ci troviamo

29. A. De Carlo, Uccelli da gabbia e da voliera, Torino, Einaudi, coll. «Nuovi Coralli», I982, quarta di copertina. 30. È quanto sostiene, a nostro avviso in modo condivisibile, S. Tani in Il romanzo di ritorno, op. cit., p. 252. E di questo sembra consapevole lo stesso De Carlo, che in più occasioni fa risalire a Macno una svolta nella sua carriera. "Sicuramente c'è stata una seconda fase che è quella di Macno e Yucatan dove ho cercato di utilizzare addirittura un linguaggio più che fotografico, a quel punto televisivo quasi», afferma nell'intervista a Cornelia Klettke (A colloquio con Andrea De Carlo, op. cit., p. I84). Mentre a Petito, nel corso di un breve esame del suo rapporto con la critica letteraria, afferma: «Con il terzo, Macno, ho conquistato un pubblico molto più vasto, e quasi per un riflesso automatico l'atteggiamento dei critici si è trasformato in ostilità" (R. Petito, Intervista inedita ad Andrea De Carlo, op. cit., p. I48).

3I. In relazione a questo tema, si leggano le pagine di C. Benussi e G. Lughi, Il romanzo d'esordio tra immaginario e mercato, Venezia, Marsilio, 1986. 
a condividere pienamente il giudizio che Asor Rosa ha dato su De Carlo nella sua recente Storia europea della letteratura italiana: «Se l'impulso ad accumulare storie e particolari e ad evocare persone fosse più sorvegliato, ne potrebbe venir fuori un'opera definitiva» ${ }^{32}$.

Questa via intrapresa, aggiungiamo senza particolari snobismi critici, che corrisponde a una scelta di intrattenere abilmente il lettore, permette, proprio a partire dagli anni Ottanta, di consolidare le strutture editoriali, e contribuisce al ritorno in primo piano della letteratura italiana, che nel decennio precedente aveva lasciato il campo alla saggistica.

È proprio questa possibile linea di ricerca narrativa, inoltre, che Calvino, attento alle dinamiche editoriali e culturali contemporanee, aveva individuato in questo decennio. Si legga in proposito l'inchiesta curata da Nico Orengo per le pagine di Tuttolibri nel 1980, in cui Calvino, interrogato su una narrativa che, negli anni Ottanta, sembrava segnata in modo sempre più evidente da esigenze commerciali, si era espresso con queste parole:

La narrativa di consumo è un fatto assolutamente negativo quando ha pretese poetiche o intellettuali o di profondità di sentimenti ecc. Quando cioè è un sottoprodotto culturale. E nella maggior parte dei casi oggi la narrativa di consumo è proprio questo: la peggiore possibile. Ma se per narrativa di consumo si intende una narrativa cosciente dell'aspetto artigiano delle proprie operazioni, dei meccanismi dei procedimenti e degli effetti, io ho pieno rispetto e ammirazione per le sue riuscite. Oggi però è la merce pseudo letteraria che vende di più, mentre della bravura nella costruzione artigiana che il romanzo richiede non si tiene nessun conto ${ }^{33}$.

Molto diverso il caso di Del Giudice. La sintonia che Calvino ha con Del Giudice è, da subito, molto forte, e si rivela prima ancora dell'esordio letterario dell'autore. Del Giudice nasce infatti come critico letterario e, come tale, ha occasione di incontrare e di intervistare Calvino, per le pagine di Paese Sera, nel 1978. In questa breve intervista, fra l'altro, Del Giudice fa prova di grande acutezza, mettendo a fuoco alcuni nuclei tematici che saranno poi essenziali negli studi critici posteriori su Calvino. E questi deve essersene accorto, se parla dell'intervista in questi termini, confrontandola ad altre: «[in] quella lì del "Resto del Carlino" non dicevo proprio niente perché l'intervistatore non mi invogliava. Invece ce n'è stata una su "Paesesera" 7 gennaio che è proprio una intervista in cui dico qualcosa, credo".

Ai fini del nostro discorso, è interessante un'immagine che viene usata da Calvino, al momento di rispondere alla domanda di Del Giudice («Per

32. A. Asor Rosa, Storia europea della letteratura italiana, Torino, Einaudi, 2009, p. 604.

33. I. Calvino, Ammiro l'artigiano che sa raccontare, quattro risposte all'inchiesta di N. Orengo, «Romanzo, se ci sei batti un colpo", Tuttolibri, I novembre i980, pp. 4-5. 
stare in un posto ne stai lontano. A Parigi, guardando l'Italia. Che prestidigitazione è questa?»):

Tra le Città invisibili ce n'è una su trampoli, e gli abitanti guardano dall'alto la propria assenza. Forse per capire chi sono devo osservare un punto nel quale potrei essere e non sto. Come un vecchio fotografo che si metta in posa davanti all'obiettivo e corra poi a schiacciare la peretta, fotografando il punto in cui poteva esserci e non c'è. Magari è questo il modo in cui i morti guardano i vivi, mescolando interesse e incomprensione ${ }^{34}$.

Del Giudice sembra quindi collocarsi subito e con naturalezza sulla linea di Calvino ${ }^{35}$, e la prova ne è un'amicizia intensa, che è passata attraverso una dichiarata attestazione di stima: "Calvino secondo me è stato lo scrittore più importante del dopoguerra, e anche lo scrittore più innovativo che ci sia stato in Italia», afferma nel corso di una sua lunga intervista nella quale misura, appunto, tutto il debito con il suo maestro, oltreché le differenze che li separano, significativamente ricondotte ad un diverso modo di intendere la descrizione:

Io però non muovo da un progressivo alleggerimento di un progetto culturale, sociale, etico complessivo, che via via ho dovuto raffinare e rendere in qualche modo adeguato. [...] Quindi non credo nella descrizione come forma di aderenza al mondo, ma credo nella descrizione come forma narrativa, perché solo la descrizione mi permette di tenere intrecciate una complessità di relazioni e di dar conto del fatto che tra osservatore e cosa osservata c'è indistinguibilità e reversibilità ${ }^{36}$.

Fra le interviste e gli interventi critici che Del Giudice dedica a Calvino ${ }^{37}$, un articolo è particolarmente interessante ai fini del nostro discorso. Si tratta della conversazione a due voci significativamente intitolata $C$ ’è ancora possibilità di narrare una storia? In essa Del Giudice sembra mettere a fuoco tutte le tematiche che potremmo definire calviniane intorno alle quali sceglierà di costruire il suo percorso. Si parla, ad esempio, dell'esperienza

34. Un altrove da cui guardare l'universo. Colloquio con Italo Calvino, a cura di D. Del Giudice, in Paese Sera, 7 gennaio 1978, apparso in Eremita a Parigi (1994), poi raccolto con il titolo Situazione 1978 in I. Calvino, Saggi. 1945-1985, op. cit., t. II, pp. 2828-2834 (la citazione è alle pp. 2832-2834). Le parole di Calvino citate in relazione all'intervista rilasciata a Del Giudice sono invece contenute in una lettera a Guido Neri del 3I gennaio 1978, in Lettere. 1940-1985, op. cit., p. 1360 .

35. "In tema di eredità, qui siamo ai massimi livelli», ha notato Asor Rosa nella sua Storia europea della letteratura italiana, op. cit., p. 59I.

36. D. Del Giudice, Il tempo del visibile nell'Atlante di Daniele Del Giudice, op. cit., pp. 84-85.

37. Oltre agli interventi già citati, si veda il colloquio con Calvino su Stendhal («Stendhal, un uomo che avrebbe voluto essere un altro", Paese Sera, I9 marzo I980, p. 3); la presenza, insieme ad Alberto Asor Rosa, Cesare Cases, Gianni Celati, Franco Fortini e Luigi Malerba, alla tavola rotonda del convegno dedicato a Calvino nel 1987: Italo Calvino. Atti del convegno internazionale, Firenze, Palazzo Medici-Riccardi, 26-28 febbraio 1987, edizione a cura di G. Falaschi, Milano, Garzanti, I988, pp. 38I-406; la partecipazione con «Un écrivain diurne» al dossier dedicato a Calvino dal Magazine littéraire, n. 274, febbraio 1990, pp. 26-29. 
scientifica come narrazione ( $\left(\grave{E}\right.$ probabile che $\mathrm{E}=\mathrm{mc}^{2}$ sia uno dei più bei racconti mai scritti», afferma); della fotografia (dopo aver citato La Chambre claire di Barthes, Del Giudice sostiene che «Nell'essenza della fotografia vi è dunque qualcosa di simile alla narrazione», mentre Calvino gli replica: «Sì, però la fotografia è al presente, un presente che ti si presenta come passato»); del viaggio, fisico e mentale; e si finisce sulla «somma dei possibili» e sulla definizione calviniana della narrativa come «escamotage all'unicità» ${ }^{3}$. $\grave{E}$ un discorso condotto da pari a pari, il cui tono è molto indicativo della natura della relazione che è maturata fra i due scrittori, e che al tempo stesso indica le vie dell'invenzione narrativa di Del Giudice, che passerà dallo scegliere uno scienziato come protagonista di Atlante occidentale, nel I985, a dedicare il suo ultimo romanzo, Orizzonte mobile, a figure di esploratori del passato e del presente.

L'allievo e il maestro si riconoscono, nel I980, anno in cui avviene questa conversazione, in una comune visione che è la risposta al diffuso interrogativo di quei tempi: è ancora possibile continuare a raccontare? E che forma può prendere la narrazione? 39

La risposta di Del Giudice cambia sempre, pur mantenendosi fedele a una precisa linea di ricerca: è quanto, pur se in modi diversi, avviene in Calvino. Del Giudice però, a differenza del suo maestro, scrive poco, in una perenne tentazione a scomparire. Dopo l'esordio, nel 1983, escono Atlante occidentale nel 1985, Nel museo di Reims nel 1988, e poi dobbiamo aspettare gli anni Novanta per le raccolte Staccando l'ombra da terra, $1994 \mathrm{e}$ Mania, 1997. A parte la parentesi del testo drammatico dedicato a Ustica, I-TIGI Canto per Ustica del 200I, si deve aspettare il 2009 per leggere un altro romanzo. La sua produzione narrativa, quindi, è rara, ridotta, pensata - molto diversa da quella di Del Carlo, alla quale sembra quindi essere apparentabile esclusivamente agli inizi. È un percorso che Del Giudice sembra quasi faticare a continuare oltre e dopo la scomparsa di Calvino, che pure ne aveva orientato la ricerca.

È il caso dell'attenzione per la scienza, ad esempio, così scarsa nella storia letteraria italiana del Novecento, ma di cui Calvino è stato un magistrale interprete, e che Del Giudice ha tenuto presente nelle pagine di Atlante

38. "C'è ancora possibilità di narrare una storia? Conversazione tra Italo Calvino e Daniele Del Giudice», Pace e guerra, n. 8, novembre i980, pp. 24-26.

39. Di un esordio narrativo di Del Giudice, tutto proteso a dare risposta all'interrogativo «È possibile oggi narrare ancora?» parla in particolare Maria Pia Ammirati, assumendo come punto di partenza l'esempio di positività portato da Calvino con il suo Se una notte d'inverno un viaggiatore, e considerando Del Giudice una sorta di corrispondente di questo esempio (M. P. Ammirati, Il vizio di scrivere. Letture su Busi, De Carlo, Del Giudice, Pazzi, Tabucchi e Tondelli, Soveria Mannelli, Rubbettino, 1991, pp. 67-75. La citazione è a p. 69). 
occidentale. E, ancora più evidente, il discorso sui Cinque sensi: la progettata raccolta di Calvino attraversa tutta la narrativa di Del Giudice, dalle pagine di Nel museo di Reims, racconto lungo del 1988 che ha al centro il tema della vista; alle pagine di Mania - tutte giocate su un «troppo sentire» di foscoliana memoria (e infatti le parole di Foscolo sono collocate in epigrafe del testo) -, volume del 1997 che si apre su un racconto dal titolo L'orecchio assoluto. Una raccolta di manie, di fissazioni e di follie, che sembrano rispondere all'ossessività che Calvino aveva individuato come una delle caratteristiche dell'agire moderno. Interrogato sulla «direzione più promettente verso cui si muove il romanzo degli anni Ottanta», egli aveva infatti risposto con precisione citando quello che per lui è il «romanzo nuovo più interessante», La vie, mode d'emploi di Georges Perec:

La mania dei puzzle di un personaggio si allarga a sistema del mondo ed è al centro di una proliferazione di storie e di avventure e descrizioni di ambienti, in cui confluiscono Borges, Roussel, Robbe-Grillet. Ma soprattutto c'è Balzac e tutta la società contemporanea in una copiosa commedia umana inaspettatamente resuscitata con le caratteristiche d'oggi, cioè lo humour nero, il senso del vuoto e la ossessività ${ }^{\circ}$.

Le sei proposte calviniane per il nuovo millennio lasciano, infine, qualcosa di più che un segno, nell'elaborazione dell'immaginario letterario di scrittori come Del Giudice. La visibilità, centro e fulcro del suo racconto e del suo modo di narrare, ma anche la leggerezza, che sembra tematizzata nella passione per gli aerei che Del Giudice, da esperienza di vita, fa passare alla sua scrittura: si veda in particolare la dichiarazione d'amore per il volo che è al centro di Staccando l'ombra da terra, ed è anticipata dalla passione che accomuna Brahe e Epstein, i due protagonisti delle pagine di Atlante occidentale.

Nel complesso, siamo davanti a un Del Giudice che cambia sempre, eppure in ogni romanzo e racconto si può capire che è lui. Potremmo quindi attribuirgli le parole con cui Calvino sembra definirsi quando, nelle pagine di Se una notte d'inverno un viaggiatore, parla dell'ineffabile figura dello scrittore: «Ti prepari a riconoscere l'inconfondibile accento dell'autore»-ammicca verso il personaggio del lettore l'io narrante dell' incipit del romanzo - «No. Non lo riconosci affatto. Ma, a pensarci bene, chi ha mai detto che questo autore ha un accento inconfondibile? Anzi, si sa che è un autore che cambia molto da libro a libro. E proprio in questi cambiamenti si riconosce che è lui» ${ }^{41}$.

40. I. Calvino, "Ammiro l'artigiano che sa raccontare», in N. Orengo (a cura di), Romanzo, se ci sei batti un colpo, op. cit., p. I5.

4I. Id., Se una notte d'inverno un viaggiatore (1979), in Romanzi e racconti, op. cit., p. 6r9. 
De Carlo, invece, non è cambiato quasi mai, dopo gli esordi. La sua produzione, continua e costante, va chiaramente verso un lettore che non vuole deludere, e del quale tiene ben presenti le aspettative.

Il Calvino «binario», come è stato definito dalla critica, sembra trovare un'incarnazione anche in questa duplicità delle sue scelte in veste di letterato-editore, e quindi nella biforcazione De Carlo-Del Giudice, che pure si presenta, nel contesto dell'Einaudi dei primissimi anni Ottanta - alla vigilia della crisi dell'azienda ${ }^{42}$ - come una proposta compatta, un'indicazione unitaria di tendenza. Un Calvino che opera, fra l'altro, all'interno della casa editrice torinese, così come Del Giudice, che dal 1986 - all'indomani della scomparsa del suo maestro - è consulente editoriale presso la stessa Casa: un'Einaudi che, salvo una breve parentesi (Nel museo di Reims esce per i tipi della Mondadori, nel 1988), fino a oggi non ha abbandonato, dimostrando una fedeltà che gli è stata riconosciuta da Giulio Einaudi stesso. E, al tempo stesso, l'autore di Se una notte d'inverno un viaggiatore non disdegna i validi "artigiani" della scrittura, che sono in questi anni in grado di andare incontro ai gusti del pubblico come sembra fare De Carlo.

Fra i meriti di Calvino, va quindi preso in considerazione anche questo: la sua capacità di intravedere, negli anni Ottanta, la doppia via che la nuova narrativa italiana avrebbe percorso negli anni successivi.

42. In proposito, si legga la testimonianza di Del Giudice, che si richiama al suo ingresso in Einaudi sia come autore che come consulente: «Il libro [Lo stadio di Wimbledon] fu pubblicato nel maggio dell'83, in ottobre ricevetti una telefonata da Alberto Papuzzi, responsabile dell'ufficio stampa, mi disse: "L'Einaudi non esiste più". Risposi: "Tutta colpa del mio libro?" Aveva ancora un certo spirito e rispose: "No, non tutta". Nei mesi successivi fu decretato il fallimento, e quando due anni dopo consegnai il secondo libro non trovai quasi nessuno, poche le persone attorno a Ernesto Ferrero. Consideravo un onore essere lì nel momento della disfatta. Nell'86 cominciai a partecipare alle riunioni del mercoledì, e per me fu una grande occasione. Ho l'impressione di aver più imparato che proposto.» (D. Del Giudice, «Senza prezzo di copertina», I quaderni di Panta, n. I5, a cura di L. Lepri, Milano, Bompiani, 200I, p. I23.) 
